\title{
Quantifying Intracardiac Organization by Combined Spectral and Spatial Phase Analysis of the Electrocardiogram
}

\author{
SM Narayan, V Bhargava \\ University of California, San Diego, USA
}

\begin{abstract}
We hypothesized that ECGs exhibiting a predominant spectral peak reflect temporal regularity, and that waves that maintain their shape between axes reflect spatial regularity, in cardiac activation. We analyzed ECGs of 38 patients at electrophysiologic study (EPS). Atrial or ventricular activity was depicted as a series of correlation values of a template to the ECG over time. In regular supraventricular or ventricular arrhythmias at invasive EPS, temporal phase was maintained since ECG spectra showed peaks $\geq 6 d B$ magnitude, and spatial phase was maintained between axes since correlation time-series, plotted for lead V5 vs aVF (XY), V5/V1 (XZ) and $a V F / V 1(Y Z)$, resulted in reproducible loops parallel to the lines of identity. Supraventricular or ventricular arrhythmias with non-uniform intracardiac spatial or temporal activation did not maintain temporal or spatial phase. Analysis was insensitive to the effects of simulated noise, baseline wander, altered gain and template selection.
\end{abstract}

\section{Introduction}

Determining whether variable ECG waveforms represent meandering intracardiac activation or biologic variability in reproducible circuits may be difficult. This is particularly true for atrial arrhythmias, whose small waveform amplitude may obfuscate the ECG diagnosis of isthmus-dependent (IDAFL) from non-isthmus dependent (NIDAFL) atrial flutter [1, 2] or even atrial fibrillation (AF) [3]. Reasoning that intracardiac regularity of atrial or ventricular arrhythmias must be reflected on the ECG, we hypothesized that careful measurement of temporal phase (regularity in timing) and spatial phase (regularity of waveform shape between axes over time) will indicate reproducible circuits, and that deviations will indicate variable circuits. We tested this hypothesis in patients undergoing invasive electrophysiologic study (EPS).

\section{Methods}

We studied 38 patients referred to the Arrhythmia service of the University of California (UCSD), and Veterans Administration (VA) Medical Centers, San Diego, for electrophysiologic study (EPS) or ablation. All studies were approved by the joint UCSD/VA Institutional Review Board for the study of Human Subjects. We recruited patients with atrial tachycardia (AT, $\mathrm{n}=4$ ), atrioventricular nodal reentry (AVNRT, $\mathrm{n}=3$ ), orthodromic reciprocating tachycardia (ORT, $\mathrm{n}=4)$, isthmus (IDAFL, $\mathrm{n}=8$ ) and non-isthmus (NIDAFL, $\mathrm{n}=6$ ) dependent atrial flutter, atrial fibrillation $(\mathrm{AF}, \mathrm{n}=3)$, monomorphic (VT-MM, n=5) and polymorphic (VT-PM, $\mathrm{n}=5$ ) ventricular tachycardia.

ECGs of the index arrhythmia were digitized at $1 \mathrm{kHz}$ and 16 bit resolution from the physiologic recorder (Bard, MA, USA. ECG analysis of temporal and spatial phase was performed using custom software written by the authors in the Labview language (National Instruments, TX, USA) running under Windows 2000 on a PC.

\subsection{Determining Temporal Phase}

First, an atrial or ventricular waveform template of 120 to $200 \mathrm{~ms}$ duration was selected from the ECG. The periodicity of this waveform in the ECG was represented as a series of its correlation values to the ECG over time. Each value was computed using the Pearson correlation coefficient on $M$ pairs of data $\left\{A_{k+i}, B_{j+i}\right\}$ from a given ECG lead, where $A_{k+i}$ is the template (of duration, $M$ data points, and ECG start position L) and $\mathrm{B}_{\mathrm{j}+\mathrm{i}}$ is the ECG sample of duration $\mathrm{M}$ selected for successive time ECG intervals, as follows:

$$
r_{j}=\frac{M\left(\sum_{i=1}^{M} A_{k+i} B_{j+i}\right)-\sum_{i=1}^{M} A_{k+i} \sum_{i=1}^{M} B_{j+i}}{\sqrt{\left[M \sum_{i=1}^{M} A_{k+i}^{2}-\left(\sum_{i=1}^{M} A_{k+i}\right)^{2}\right]\left[M \sum_{i=1}^{M} B_{j+i}^{2}-\left(\sum_{i=1}^{M} B_{j+i}\right)^{2}\right]}}
$$

where $r_{j}$, is the correlation coefficient value at the $\mathrm{j}^{\text {th }}$ 
ECG time point, $1 \leq \mathrm{j} \leq \mathrm{Q}-\mathrm{M}-1$, Q is the last ECG point; also $\mathrm{L} \leq k \leq \mathrm{L}+\mathrm{M}-1$, $\mathrm{L}$ is the first fixed template point, $1 \leq \mathrm{L} \leq \mathrm{Q}-\mathrm{M}$. Fig. 1 shows the correlation time series using an atrial template in a case of isthmus dependent Atrial Flutter.

Maintenance of temporal phase, reflecting precise regularity in intracardiac timing, should produce a dominant spectral peak. We therefore computed Fast Fourier Transforms on 8.192 seconds ( $2^{13}$ data points) of each ECG, as well as on the time series of correlation values of the template to its ECG.

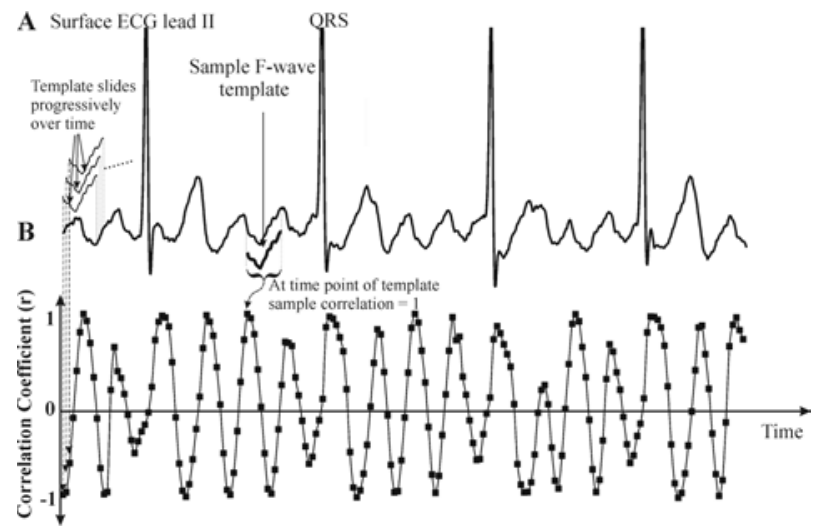

Figure 1. F-wave template slides across ECG to yield a correlation time series where $\mathrm{r} \approx 1$ indicates $\mathrm{F}$-wave recurrence.

Fig. 2 shows a typical ECG spectrum with its atrial and ventricular components (fig. 2A), and the predominantly atrial components in correlation spectra (fig. 2B) in a case of IDAFL.
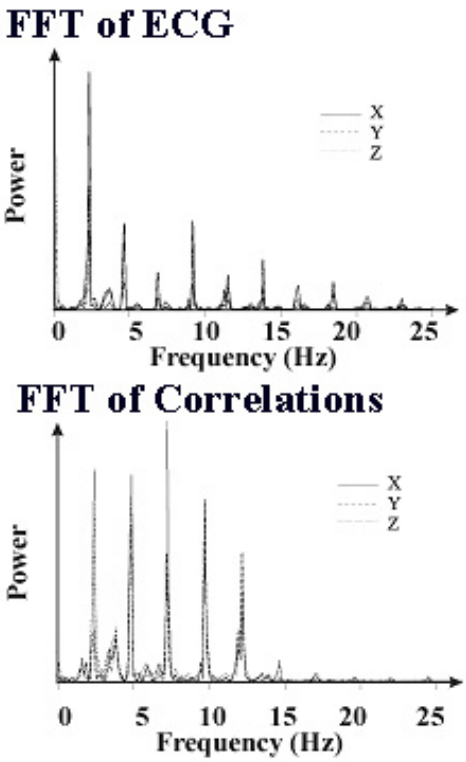

Figure 2. In the case of AT (from fig. 1), A. ECG Spectrum and B. Correlation Spectrum shows discrete atrial and ventricular components, subharmonics and harmonics.
Temporal phase maintenance (coherence) was defined as atrial peak magnitude $\geq 6 \mathrm{~dB}$ compared to 2 frequency points either side, from ECG or correlation spectra, and is demonstrated in both figs $2 \mathrm{~A}$ and $2 \mathrm{~B}$ for AT.

\subsection{Determining Spatial Phase}

Spatial phase in the $\mathrm{XY}$ plane was computed by plotting $X$ - versus $Y$ - correlation values at each time point; similarly for $\mathrm{YZ}$ and $\mathrm{XZ}$ planes. The resulting loops (fig. 3) for AT show that spatial phase was maintained (coherent) since correlations maintain a fixed relationship between axes over time, i.e. loops reached the $(1,1)$ coordinate, reflecting simultaneous recurrence of the template $(r=1)$ in each axis, and were reproducible.

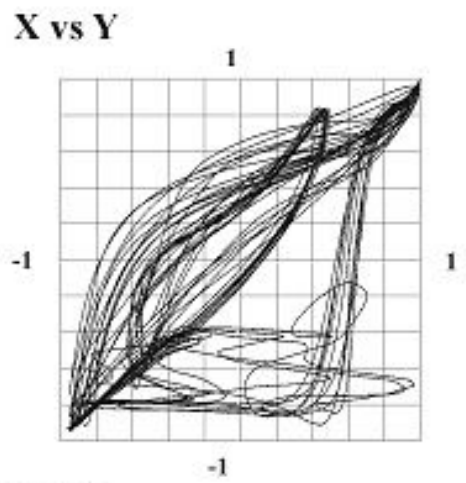

Yvs Z

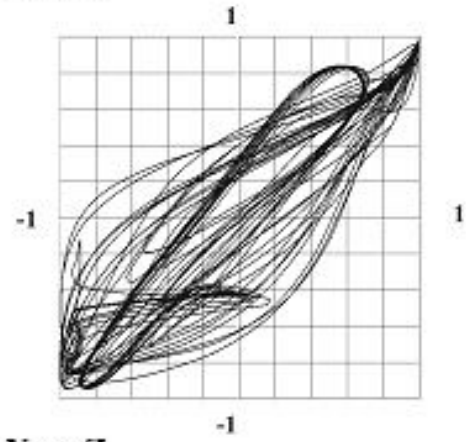

$\mathrm{X}$ vs Z

1

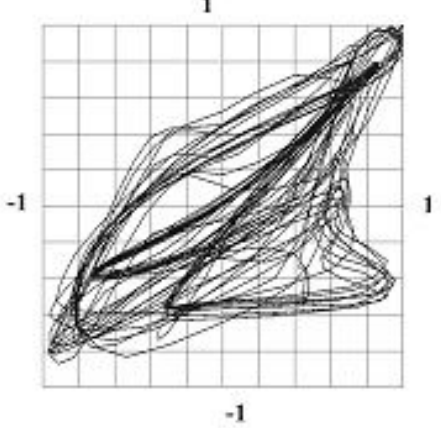

Figure 3. Spatial Correlation Plots (XY, YZ, XZ) showing reproducible loops approaching the $(1,1)$ point in each plane in the case of AT shown above. 


\subsection{Simulations}

We tested the behavior of phase analysis in response to simulated noise, baseline wander, alterations in gain and template selection.

\section{Results}

\begin{tabular}{ccc}
\hline Rhythm & $\begin{array}{c}\text { Temporal } \\
\text { Coherence }\end{array}$ & $\begin{array}{c}\text { Spatial } \\
\text { Coherence }\end{array}$ \\
\hline AT $(n=4)$ & $4 / 4$ & $4 / 4$ \\
AVNRT $(n=3)$ & $3 / 3$ & $3 / 3$ \\
ORT $(n=4)$ & $4 / 4$ & $4 / 4$ \\
IDAFL $(n=8)$ & $8 / 8$ & $8 / 8$ \\
NIDAFL $(n=6)$ & $6 / 6$ & $1 / 6$ \\
AF $(n=3)$ & $0 / 3$ & $0 / 3$ \\
VT-MM $(n=5)$ & $5 / 5$ & $5 / 5$ \\
VT-PM $(n=5)$ & $0 / 5$ & $0 / 5$ \\
\hline
\end{tabular}

\subsection{Temporal Phase}

Temporal phase (spectral peak $\geq 6 \mathrm{~dB}$ ) was maintained in arrhythmias exhibiting regular intracardiac activation, including AT, AVNRT, ORT, IDAFL and NIDAFL, as well as VT-MM. Conversely, temporal phase was not maintained in AF and VT-PM. Fig 4 shows a typical ECG spectrum for AF, showing broadband spectra without a dominant peak. Correlation spectra (to several putative 'F-waves') were similarly broadband.

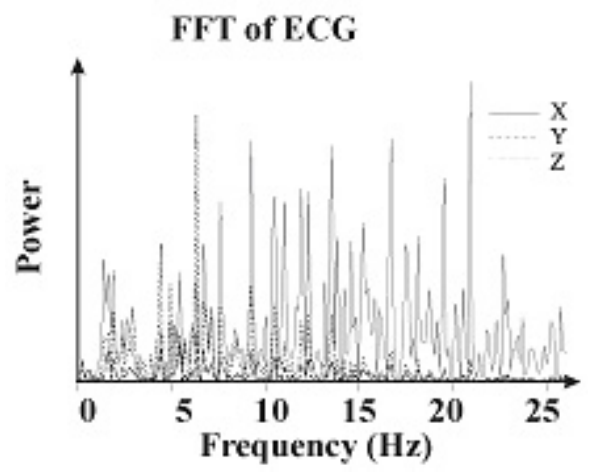

Figure 4. ECG Spectra in AF showing Lack of temporal coherence with broadband spectra $(>10 \mathrm{~dB})$ and no predominant peak. Similar spectra were seen in VT-PM.

\subsection{Spatial Phase}

Spatial phase was maintained (coherent) in AT, AVNRT, ORT, IDAFL and VT-MM, evidenced by reproducible spatial loops that approach the $(1,1)$ coordinate in all planes. Conversely, spatial phase was not maintained in AF and VT-PM, shown by the space filling, irregular loops in fig 5. Surprisingly, the loops of
NIDAFL deviated from reproducibility, although less than AF and VT-PM.
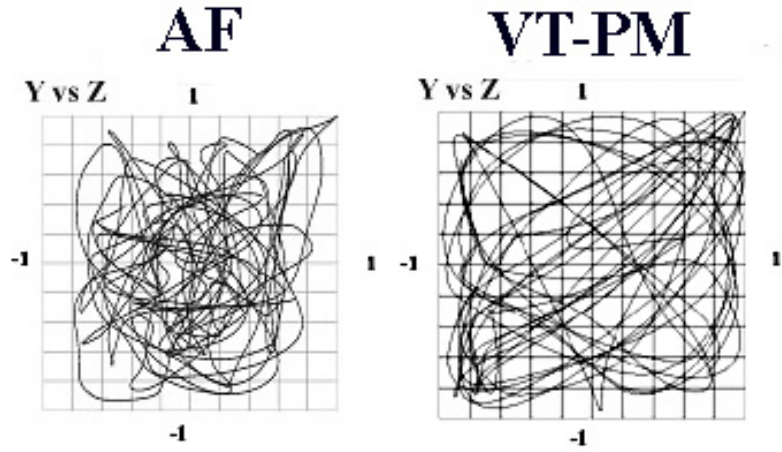

Figure 5. Spatial Correlation Plots of Left, AF and Right, VT$\mathrm{PM}$, showing irregular and non-reproducible activity between planes (YZ shown). This indicates that successive cycles trace different spatial vectors.

\subsection{Effects of Noise on Analysis}

Spatial and temporal phase analysis were insensitive to (a) Baseline noise of $10 \%$ of root-mean-square (fig. 6 , shown for IDAFL and VT-MM), (b) baseline wander; (c) continuous or abrupt changes in gain; or (d) template choice (fig. 7, shown for IDAFL).

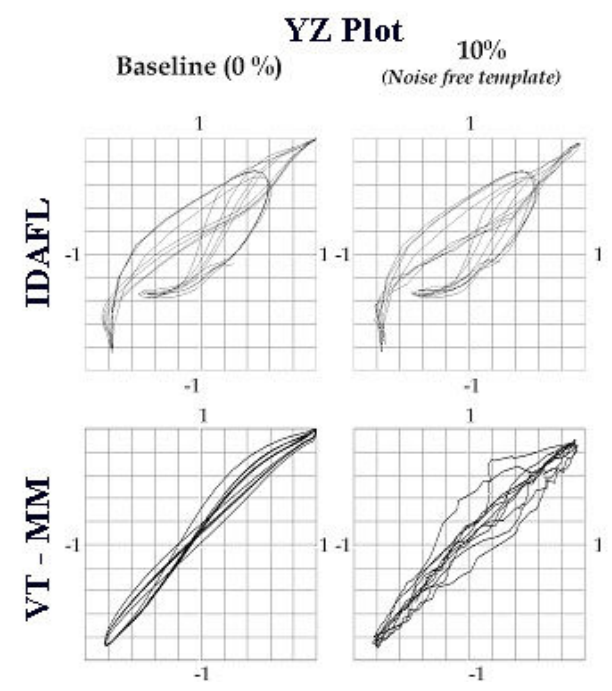

Figure 6. Baseline Noise (10\% RMS) has little qualitative effect on YZ Spatial Correlation loops in (top) IDAFL and (bottom) VT-MM 


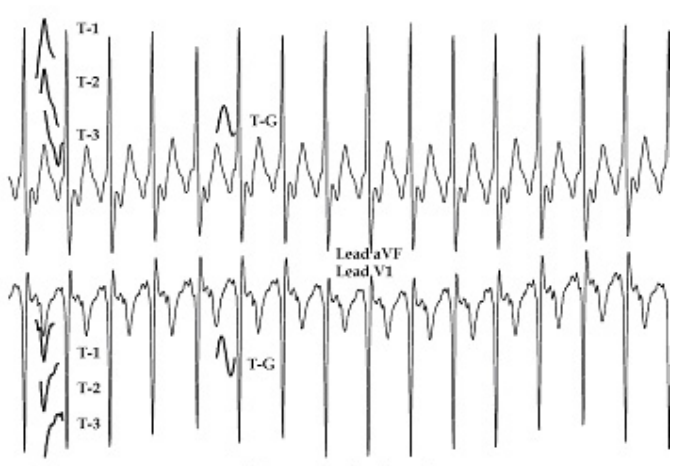

r (Correlation) Plot

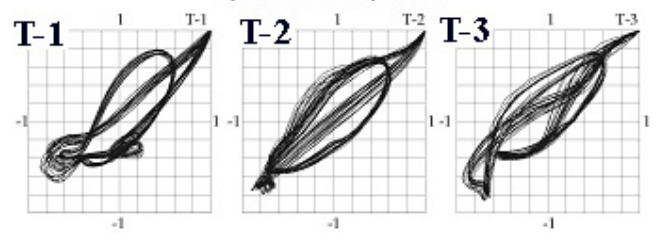

Figure 7. Specific Choice of Template (T-1, 2, 3) has little effect on YZ Spatial Correlation loops in this case of IDAFL: all result in reproducible loops that reach $(1,1)$.

\section{Discussion}

This study provides proof-of-concept that the spatial organization of intra-atrial and intra-ventricular activation can be numerically stratified from the ECG by analyzing the extent to which ECG waveforms maintain a constant (coherent) temporal and spatial relationship. In this pilot clinical study, temporospatial coherence identified the uniform intracardiac wavefronts of SVT (including atrial tachycardia, AVNRT, ORT), IDAFL and monomorphic VT. Surprisingly, deviations from temporospatial coherence identified NIDAFL and placed it between IDAFL and 'organized' $A F$ on an organizational spectrum. AF and VF both showed a complete absence of temporal or spatial coherence.

Before concluding that the present analysis can detect subtle variations in intracardiac activation, several technical points must be considered. First, we assume that cardiac potentials map consistently to the body surface; this is true for hundreds of beats in humans [4] and several minutes in canine heart-human torso models [5]. Second, we show that several putative sources of 'noise' do not artifactually introduce or mask coherence: this method was resilient to random noise (fig. 6), varying templates (fig. 7), baseline wander and gain changes (not shown). Third, the fact that spectra and spatial loops segregated based on EPS-demonstrated uniformity suggests that our results do not predominantly reflect (random) noise. This work may thus complement uses of the ECG to detect subtle variations in atrial and ventricular activation and autonomic tone $[4,6]$.

\section{Conclusions}

Temporospatial phase analysis quantifies the temporal and spatial organization of atrial or ventricular activity from the ECG and reveals a spectrum ranging, in the atrium, from SVT and IDAFL, to NIDAFL then AF, and, in the ventricle, from VT to VF. The method is relatively insensitive to noise, and resilient to variations in its initial conditions. This analysis may help improve the ECG diagnosis of complex arrhythmias and guide the approach to ablation or response to pharmacologic therapy.

\section{Acknowledgements}

Supported in part by grant HL70529 from the National Heart, Lung and Brain Institute to SMN.

\section{References}

P. Milliez, A. W. Richardson, O. Obioha-Ngwu, P. J. Zimetbaum, P. Papageorgiou, and M. E. Josephson, "Variable electrocardiographic characteristics of isthmus-dependent atrial flutter," J Am Coll Cardiol, 2002 vol. 40, pp. 1125-1132.

[2] J. E. Marine, V. J. Korley, O. Obioha-Ngwu, J. Chen, P. Zimetbaum, P. Papageorgiou, P. Milliez, and M. E. Josephson, "Different Patterns of Interatrial Conduction in Clockwise and Counterclockwise Atrial Flutter," Circulation, 2001 vol. 104, pp. 1153-1157.

[3] J. M. Kalman, J. E. Olgin, L. A. Saxon, R. J. Lee, M. M. Scheinman, and M. D. Lesh, "Electrocardiographic and electrophysiologic characterization of atypical atrial flutter in man: Use of activation and entrainment mapping and implications for catheter ablation.," $J$. Cardiovasc Electrophysiol., 1997 vol. 8, pp. 121-144.

[4] N. G. Kavesh, M. E. Cain, H. D. Ambos, and R. M. Arthur, "Enhanced detection of distinguishing features in signal-averaged electrocardiograms from patients with ventricular tachycardia by combined spatial and spectral analyses of entire cardiac cycle.," Circulation, 1994 vol. 90 , pp. 254-263.

[5] R. N. Ghanem, J. E. Burnes, A. L. Waldo, and Y. Rudy, "Imaging Dispersion of Myocardial Repolarization, II: Noninvasive Reconstruction of Epicardial," Circulation, 2001, 104: 1306-1312.

[6] S. M. Narayan and M. E. Cain, "Non-Invasive Techniques for Assessing Arrhythmia Risk: T-Wave Alternans, Signal-Averaged ECG, and Heart Rate Variability," in Harrisons Online Textbook of Medicine, 15th Edition, 2003.

Address for correspondence:

Sanjiv M. Narayan, MB, MD, MRCP

Director, Electrophysiology Program

VA Medical Center and University of California

3350 La Jolla Village Drive

San Diego, California 92161

snarayan@ucsd.edu 